\title{
Modelling signal platoon patterns by approach lane use and movement class
}

\author{
R. Akçelik \\ Akcelik \& Associates Pty Ltd (SIDRA SOLUTIONS), Australia
}

\begin{abstract}
A lane-based signal platoon progression model has been developed for estimating performance measures as a function of signal offsets, geometric design and flow conditions. This is part of a lane-based network model that involves the blockage of upstream intersection lanes by downstream queues (backward spread of congestion) and a capacity constraint applied to oversaturated upstream intersections. The model takes into account midblock lane changes that apply to signal platoon patterns. This is particularly important in evaluating closely-spaced intersections with high demand flows where vehicles have limited opportunities for lane changes between intersections. The modelling of signal platoon patterns is further enhanced by assigning two types of movements negotiating the network to special movement classes. These are the through movements at external approaches which become turning movements at downstream internal approaches, and the dogleg movements at staggered $\mathrm{T}$ intersections. These movements can be assigned to separate lanes and separate signal phases, and their second-by-second platoon patterns can be tracked through the network separately. This improves the quality of signal platoon modelling and is expected to produce better results in assessing signal coordination quality and optimising signal offsets. The use of special movement classes also helps to estimate unequal lane use cases at external approaches of a paired intersection system, a factor which also affects signal platoon patterns. A staggered T-intersection example is presented to demonstrate important aspects of modelling signal platoon patterns by approach lane use and movement class.
\end{abstract}

Keywords: SIDRA INTERSECTION, traffic signals, intersection, network, signal coordination, movement classes, congestion, queue spillback, delay, queue, stops. 


\section{Introduction}

This paper discusses a new analytical lane-based method for determining platoon patterns at closely-spaced signalised intersections developed for the SIDRA INTERSECTION software [1-3].

Discussion of the lane-based network model in relation to modelling of lane blockage (queue spillback) effects has been presented in previous papers by the author [4-6]. This paper discusses the implications of using a lane-based method for the modelling of signal platoons.

In particular, the paper discusses the use of Special Movement Classes to assign selected movements to separate lanes and separate signal phases so as to increase the quality of signal coordination model.

A staggered T-intersection example is presented to demonstrate important aspects of modelling signal platoon patterns by approach lane use and movement class.

\section{Lane-based network model}

Unlike traditional network models that use aggregate models of links or lane groups, the lane-based network model can provide information about upstream departure and downstream arrival patterns, queue lengths, lane blockage probabilities, backward spread of queues, proportion of traffic arriving during green, and so on at an individual lane level. These are important in modelling signal platoon patterns for estimating performance measures (delay, back of queue, stop rate), and particularly important in evaluating closely-spaced intersections with high demand flows where vehicles have limited opportunities for lane changing between intersections.

The new lane-based signal platoon derives second-by-second downstream arrival patterns in accordance with the above requirements. Modelling of departure patterns at upstream lanes takes into account (i) probabilities of blockage by downstream queues and the resulting capacity reductions at blocked upstream lanes, (ii) capacity constraint at oversaturated upstream lanes resulting in reduced downstream arrival flows, and (iii) lane choices of movements from approach lanes to exit lanes at the upstream intersection (lane movement flow proportions). The modelling of arrival patterns at downstream approach lanes takes into account implied midblock lane changes.

While estimation of individual lane capacities, lane flows and lane queues is important in assessing performance of a single intersection [7-11], this becomes even more important in network modelling. The backward spread of congestion and upstream capacity constraint makes downstream and upstream lane departure and arrival patterns, lane capacities, lane flows and lane queues highly interdependent especially in the case of closely-spaced intersections.

Traditional network models have been concerned more about modelling forward movement of vehicle platoons than backward spread of queues between intersections (queue spillback) and capacity constraint (demand starvation) related to oversaturated intersection conditions [12]. Although all these elements 
are important, the lack of modelling of the capacity-reducing effect of blockage of departures by downstream queues and capacity constraint for oversaturated conditions cannot provide a satisfactory network model for the high traffic demand conditions experienced in more recent times.

\section{Signal platoon model}

The modelling of platoon arrival patterns at downstream lanes takes into account lane changes due to exit short lanes at upstream locations and approach short lanes at downstream locations, as well as midblock lane changes based on matching of upstream and downstream lane flow rates. The second-by-second upstream departure flow patterns are moved forward towards the downstream lane stop lines at the approach cruise speed while applying any required lane changes. Any midblock inflow and outflow rates are also taken into account. The method is applied by movement class (light vehicles, heavy vehicles, buses, large trucks, etc.) since each class can have a different approach cruise speed and different lane use.

The second-by-second platoon arrival patterns determined by the program are used to calculate Percent Arriving During Green, Platoon Ratio, and Delay and Queue Progression Factors for each approach lane for use in performance calculations. The method has its origin in the US Highway Capacity Manual [13] for delay calculations (using the Delay Progression Factor), and its extension by the author $[14,15]$ for back of queue, queue clearance time, proportion queued, queue move-up rate and effective stop rate calculations (using the Queue Progression Factor).

The modelling of lane-based signal platoon patterns is further enhanced by using the movement class facility of the SIDRA INTERSECTION software. This is implemented by assigning two types of movements negotiating the network to special movement classes: (i) through movements at external approaches which become turning movements at downstream internal approaches, and (ii) dogleg movements at staggered $\mathrm{T}$ intersections.

These movements can be assigned to separate lanes and separate signal phases, and their second-by-second platoon patterns can be tracked through the network separately. This improves the quality of signal platoon modelling and is expected to produce better results in assessing signal coordination quality and optimising signal offsets. The use of special movement classes also helps to estimate unequal lane use cases at external approaches of a paired intersection system, a factor which also affects signal platoon patterns.

\section{Example}

A case of signalised staggered $\mathrm{T}$ intersections with $200 \mathrm{~m}$ distance between them is considered as an example to demonstrate important aspects of modelling signal platoon patterns by approach lane use and movement class. The intersection geometry, signal phasing and related parameter values are shown in 
Figure 1. Site origin-destination flows (intersection turning volumes) and network origin-destination flows are shown in Figure 2.

The Site OD flows between the two intersections have been matched perfectly for the purpose of this analysis. If the Site OD flows at the two intersections result in upstream and downstream flows which do not match, midblock inflow and outflow values determined by the model are included in the analysis. This does not apply in this example. The Network OD flows that match the Site OD flows are also provided for analysing differences between analysis scenarios with and without the knowledge of Network OD flows.

Two analysis scenarios are considered to investigate the differences between the network model results including signal platooning and the resulting performance estimates with and without the use of special movement classes. The characteristics of the two analysis scenarios are summarised in Table 1.

Table 1: Two analysis scenarios for the example of staggered $\mathrm{T}$ intersections.

\begin{tabular}{|c|l|l|l|}
\hline $\begin{array}{l}\text { Analysis } \\
\text { scenario }\end{array}$ & $\begin{array}{l}\text { Special movement } \\
\text { classes }\end{array}$ & $\begin{array}{l}\text { Lane use of approach lanes } \\
\text { (except internal approach left turn } \\
\text { lanes) }\end{array}$ & $\begin{array}{l}\text { Midblock lane } \\
\text { changes on internal } \\
\text { approaches }\end{array}$ \\
\hline (i) & No & Equal lane use & Yes \\
\hline (ii) & Yes* & $\begin{array}{l}\text { Equal lane use except east Lanes } \\
\text { 3 and 4 (right turn) with special } \\
\text { MCs in Lane 3 }\end{array}$ & Yes \\
\hline
\end{tabular}

*Network origin - destination flows are known.

Analysis Scenario (i) assumes that only the Site OD flows (intersection turning volumes) are known at each intersection, and the Network OD flows are not known. Analysis Scenario (ii) assumes that the Network OD flows are known in addition to the Site OD flows as shown in Figure 2, and assigns special movement classes to upstream and downstream lanes according to destinations at the downstream intersection.

For all lanes, both scenarios use default lane movement flow proportions based on $100 \%$ flow to the most direct exit lane.

In all cases, equal lane use is assumed for the program to determine lane flow rates for multiple approach lanes, i.e. there are no user-specified lane utilisation ratios. Equal lane use for approach lanes means that lane degrees of saturation are equal [9-11]. Where lanes have equal capacity values, equal lane use means equal lane flows. The assumption of equal lane use for all approaches results in implied midblock lane changes. These are identified by comparing the upstream lane flows (flows at entry to an internal approach) with downstream approach (stop line) lane flows as shown in Figures 3 and 4. Movements belonging to special movement classes are shown in Figure 4.

Many other analysis scenarios are possible considering different lane use patterns and lane movement flow proportions. 


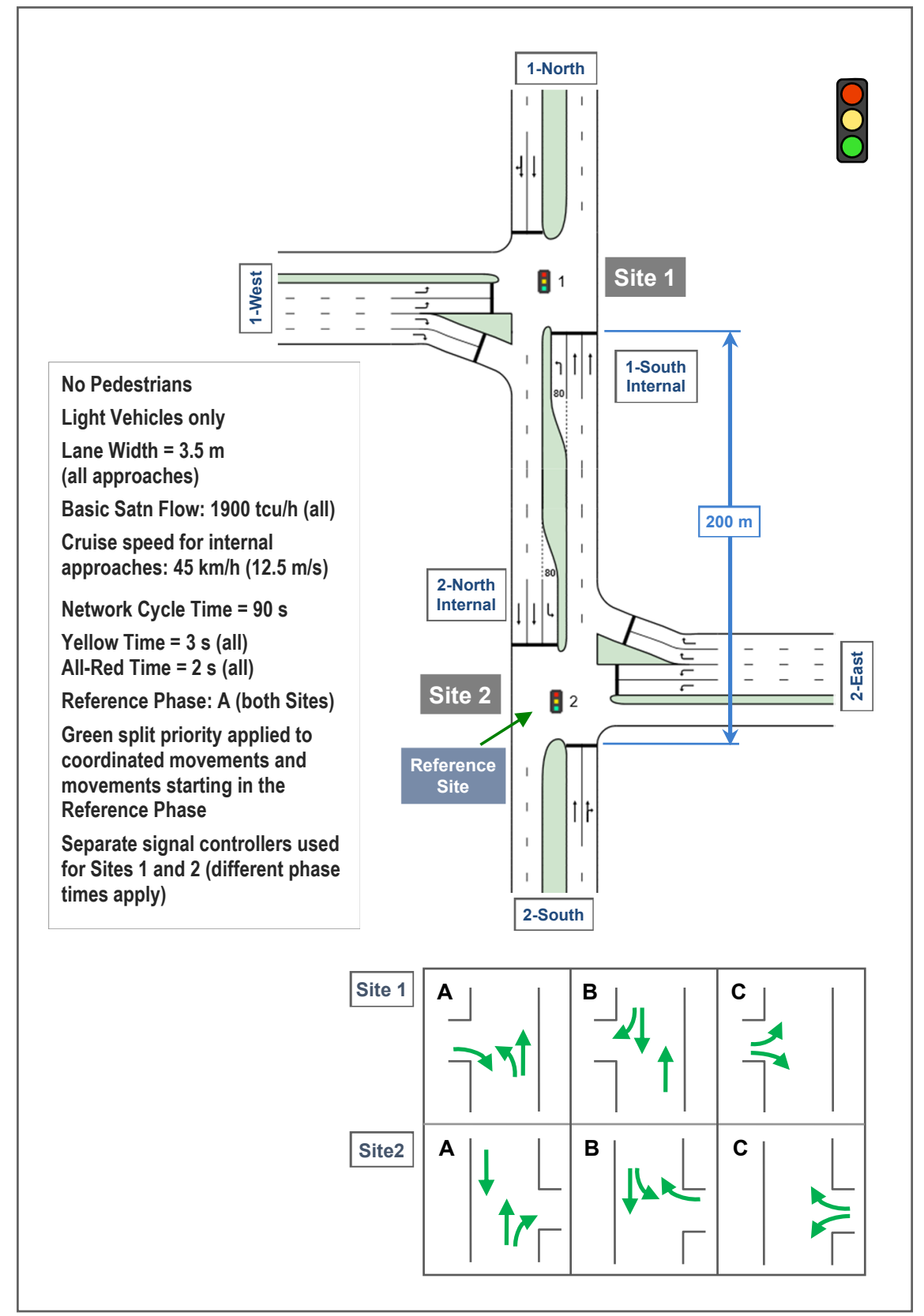

Figure 1: Example: signalised staggered $\mathrm{T}$ intersections. 


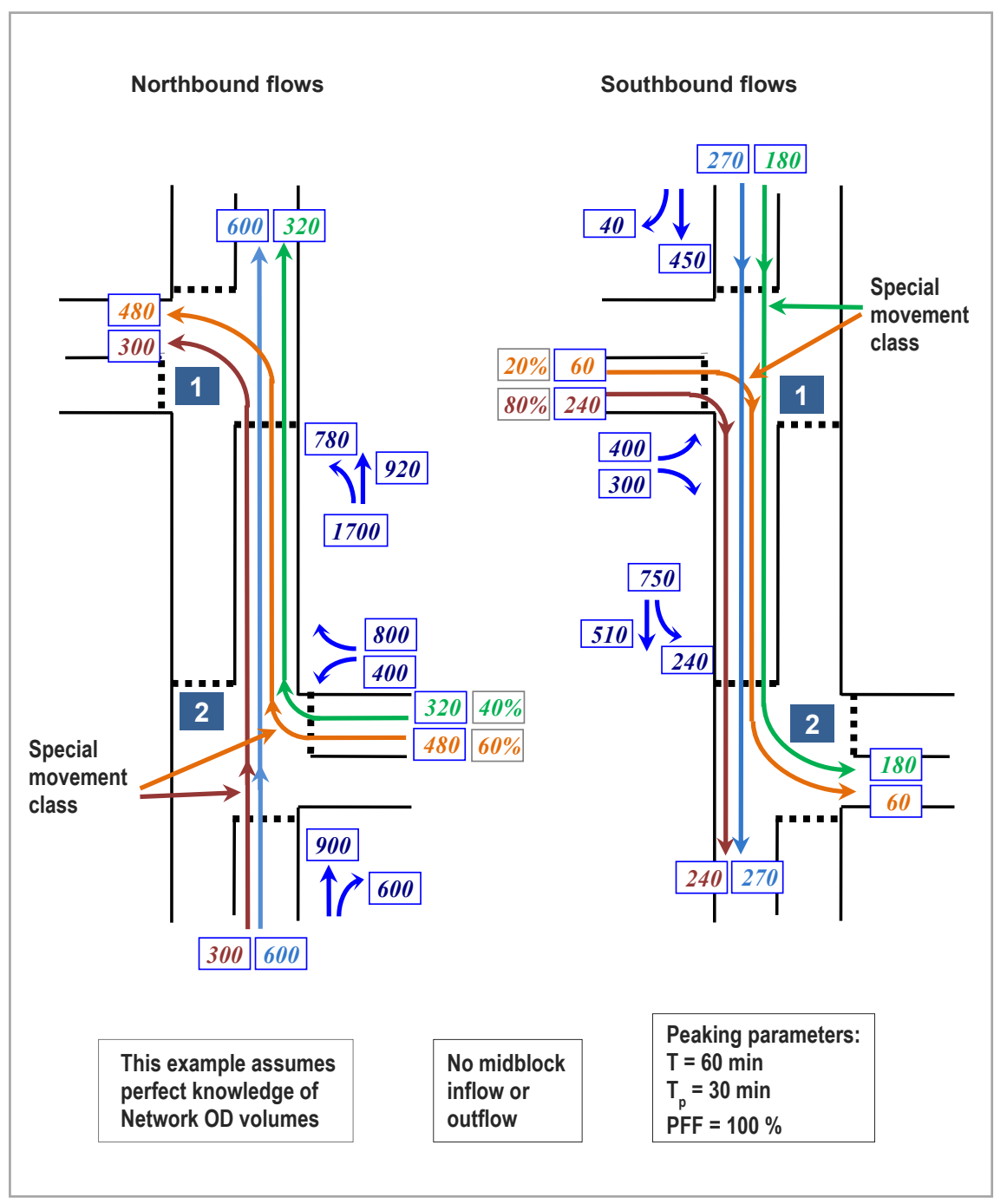

Figure 2: Site and network origin-destination flows for the example shown in Figure 1. 


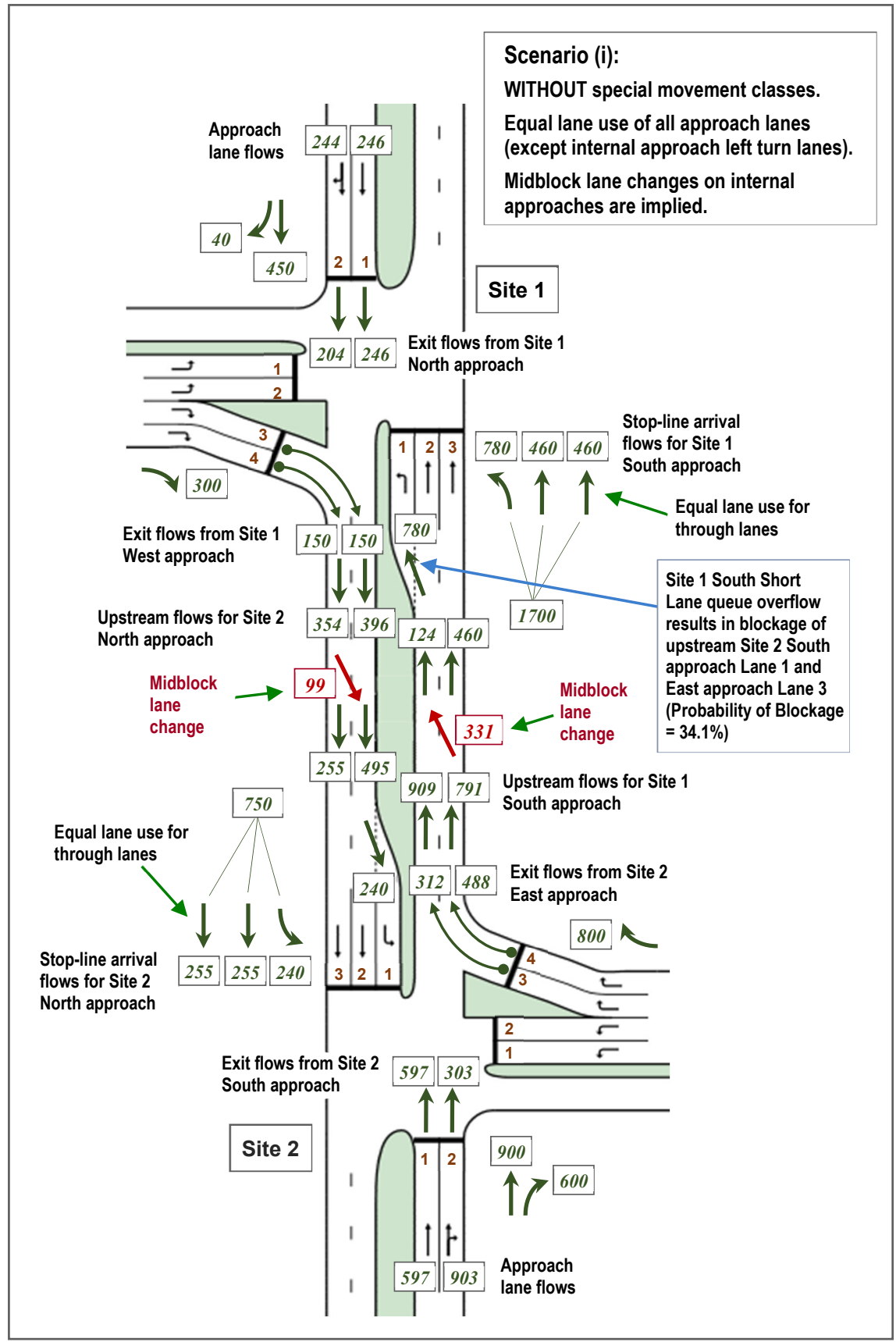

Figure 3: Scenario (i): WITHOUT special movement classes for the example shown in Figures 1 and 2. 


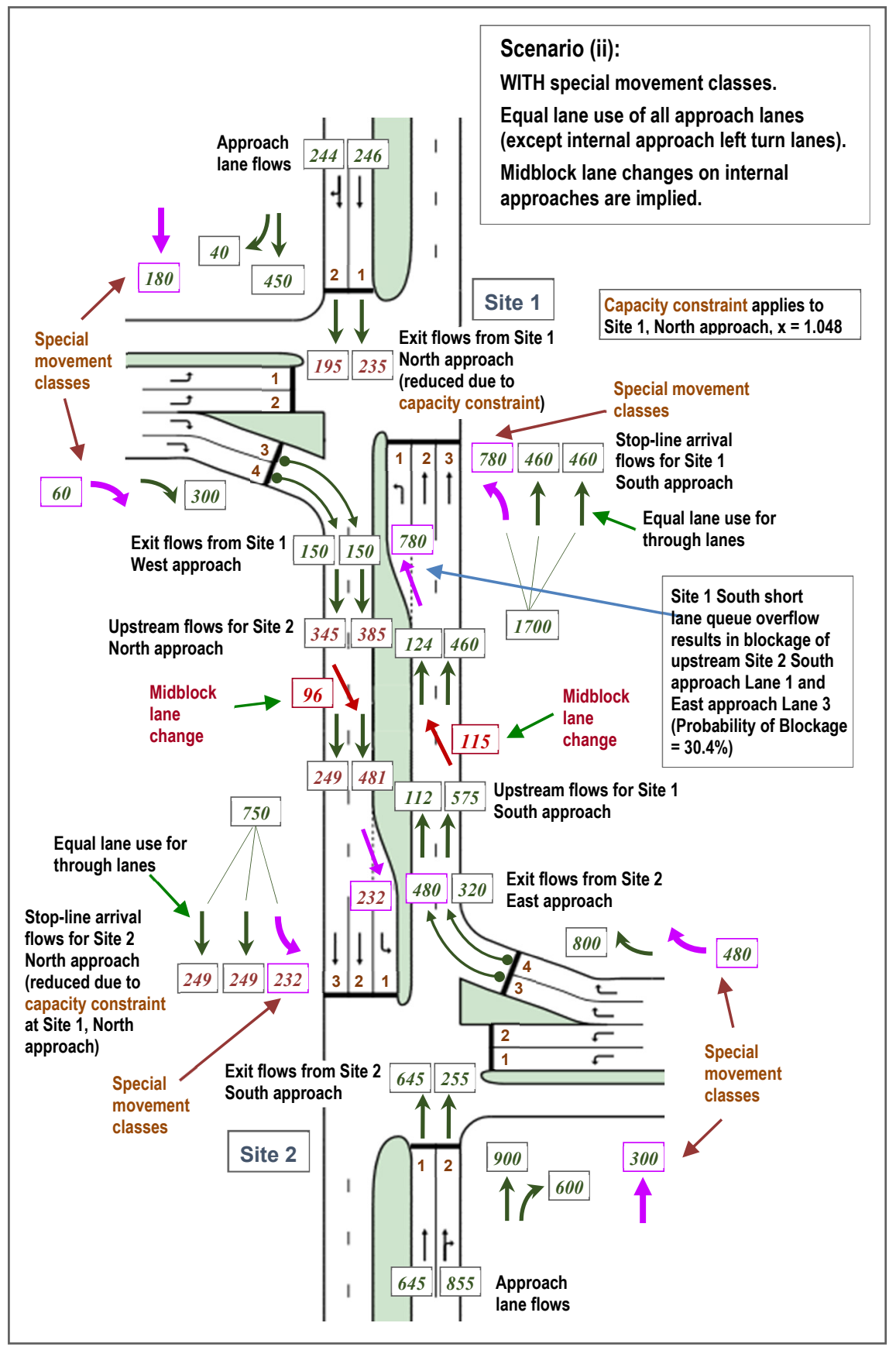

Figure 4: Scenario (ii): WITH special movement classes for the example shown in Figures 1 and 2. 
Both scenarios were analysed using a Network Cycle Time of 90 s. Phase times were calculated applying "green split priority" to coordinated (internal approach) movements as well as the external approach movements that start at the reference phase $[8,16]$. The phase times for the two scenarios differed (up to 4 seconds) due to different saturation flow rates resulting from changed movement flow rates in shared lanes and different capacity losses due to lane blockage.

For signal coordination purposes, Site 2 is the reference site (offset $=0$ ), and Phase A is the reference phase for both sites. For all scenarios, offset $=16 \mathrm{~s}$ was specified for Site 2. This means that the green time for the Site 2 Through phase starts at 0 seconds and the green time for the Site 1 through phase starts at 16 seconds. This is the travel time offset for the northbound through movement allowing for early arrival of the front of platoon due to platoon dispersion.

\section{Analysis results}

In addition to the difference between signal platoon characteristics with and without the use of special movement classes, analysis results for this example demonstrate the effects of external approach lane underutilisation, lane blockage of upstream lanes because of downstream short lane overflow, and capacity constraint.

The upstream exit and downstream approach lane flows and implied midblock lane changes for analysis scenarios (i) and (ii) are shown in Figures 3 and 4. Approach and exit lanes are numbered from left to right in the direction of movement. The lane change values for entry into short lanes differ when arrival flows are subject to upstream capacity constraint.

Comparisons of results for Scenarios (i) and (ii) for south (internal) approach lanes of Site 1 and East (external) approach lanes of Site 2 are presented in Table 2. Comparisons of results for Scenarios (i) and (ii) for internal left and through (platooned) movements for Site 1 are presented in Table 3.

The results given in Tables 2 and 3 show that there are significant differences in platoon characteristics between Analysis Scenarios (i) and (ii) as indicated by the values of percent arriving during green and platoon ratio modelled per lane and per movement.

Although the performance estimates for different analysis scenarios look close in Tables 2 and 3, the differences in individual lane values can be significant especially for the back of queue estimates when (i) the approach (midblock) distance between intersections is small, and therefore lane blockage effects are likely to come in, and (ii) when sensitivities are higher at high degrees of saturation. Average delay values per movement can hide larger values of delay in individual lanes used by the movement when there is significant unequal lane use. 
Table 2: $\quad$ Results for Scenarios (i) and (ii) for South (internal) approach lanes of Site 1 and east (external) approach lanes of Site 2.

\begin{tabular}{|c|c|c|c|c|c|c|c|}
\hline $\begin{array}{l}\text { Approach } \\
\text { lane }\end{array}$ & $\begin{array}{l}\text { Arrival } \\
\text { flow } \\
\text { (veh/h) }\end{array}$ & $\begin{array}{l}\text { Capacity } \\
\text { (veh/h) }\end{array}$ & $\begin{array}{l}\text { Degree } \\
\text { of } \\
\text { saturation } \\
(\mathrm{V} / \mathrm{C})\end{array}$ & $\begin{array}{l}\text { Per cent } \\
\text { arriving during } \\
\text { green } \\
(\%)\end{array}$ & $\begin{array}{l}\text { Platoon } \\
\text { ratio }\end{array}$ & $\begin{array}{c}\text { Average } \\
\text { delay } \\
\text { (s) }\end{array}$ & $\begin{array}{c}\text { 95th \%ile } \\
\text { back of } \\
\text { queue } \\
(\mathrm{m})\end{array}$ \\
\hline \multicolumn{8}{|c|}{ Analysis Scenario (i) WITHOUT special movement classes: Site 1 south approach } \\
\hline Lane 1 & 780 & 831 & 0.939 & $57.1 \%$ & 1.007 & 47.1 & 281 \\
\hline Lane 2 & 460 & 1472 & 0.313 & $78.5 \%$ & 1.024 & 3.1 & 40 \\
\hline Lane 3 & 460 & 1472 & 0.313 & $66.0 \%$ & 0.869 & 4.5 & 59 \\
\hline \multicolumn{8}{|c|}{ Analysis Scenario (i) WITHOUT special movement classes: Site 2 east approach } \\
\hline Lane 3 & 312 & 390 & 0.801 & $33.3 \%$ & 1.000 & 42.1 & 101 \\
\hline Lane 4 & 488 & 609 & 0.801 & $33.3 \%$ & 1.000 & 39.0 & 149 \\
\hline \multicolumn{8}{|c|}{ Analysis Scenario (ii) WITH special movement classes: Site 1 south approach } \\
\hline Lane 1 & 780 & 867 & 0.900 & $38.5 \%$ & 0.641 & 36.4 & 255 \\
\hline Lane 2 & 460 & 1493 & 0.308 & $97.1 \%$ & 1.248 & 0.8 & 6 \\
\hline Lane 3 & 460 & 1493 & 0.308 & $88.2 \%$ & 1.134 & 3.2 & 23 \\
\hline \multicolumn{8}{|c|}{ Analysis Scenario (i) WITH special movement classes: Site 2 east approach } \\
\hline Lane 3 & 480 & 503 & 0.954 & $37.8 \%$ & 1.000 & 68.7 & 215 \\
\hline Lane 4 & $320 *$ & 691 & $0.463 *$ & $37.8 \%$ & 1.000 & 28.4 & 75 \\
\hline
\end{tabular}

*Lane underutilisation.

Table 3: Comparison of results for internal left and through (platooned) movements on Site 1 south internal (northbound) approach.

\begin{tabular}{|c|c|c|c|c|c|c|}
\hline Movement & $\begin{array}{l}\text { Arrival } \\
\text { Flow } \\
\text { (veh/h) }\end{array}$ & $\begin{array}{l}\text { Degree } \\
\text { of } \\
\text { Saturation } \\
(\mathrm{v} / \mathrm{c})\end{array}$ & $\begin{array}{c}\text { Percent } \\
\text { Arriving } \\
\text { During Green } \\
(\%)\end{array}$ & $\begin{array}{c}\text { Platoon } \\
\text { Ratio }\end{array}$ & $\begin{array}{c}\text { Average } \\
\text { Delay } \\
\text { (s) }\end{array}$ & $\begin{array}{c}\text { 95th \%ile } \\
\text { Back of } \\
\text { Queue } \\
\text { (m) }\end{array}$ \\
\hline \multicolumn{7}{|c|}{ Analysis Scenario (i) WITHOUT special movement classes: Site 1 South approach } \\
\hline Left & 780 & 0.939 & $57.1 \%$ & 1.007 & 47.1 & 281 \\
\hline Through & 920 & 0.313 & $72.6 \%$ & 0.947 & 3.8 & 59 \\
\hline \multicolumn{7}{|c|}{ Analysis Scenario (ii) WITH special movement classes: Site 1 South approach } \\
\hline Left & 780 & 0.900 & $38.5 \%$ & 0.641 & 36.0 & 255 \\
\hline Through & 920 & 0.308 & $92.7 \%$ & 1.191 & 1.2 & 23 \\
\hline
\end{tabular}

The midblock lane change flow rate for Northbound movements is significantly smaller for Scenario (ii) as seen in Figures 3 and 4, indicating that the use of special movement classes provides better estimates of lane use on 
external approaches according to downstream destinations. Thus, the use of special movement classes helps with better estimation of the unequal lane use often observed at external approach lanes of closely-spaced intersections due to the network origin - destination effects. Signal timings get affected by unequal lane use, and these in turn affect platoon characteristics, delay and queue length results.

In this example, southbound platoon movements are affected by capacity constraint due oversaturated lanes on the north approach of Site 1 under Analysis Scenario (ii). This results in reduced arrival flow rates for movements on the North approach of Site 2. This is not observed under Analysis Scenario (i).

Under both scenarios, short lane queue overflows from Lane 1 of Site 1 South approach, and results in the adjacent lane queue blocking Lane 1 of the South approach and Lane 3 of the East approach of Site 2, thus causing capacity reductions on these lanes. The amount of blockage is different under Analysis Scenarios (i) and (ii).

\section{Conclusions}

The lane-based analytical network model used in the SIDRA INTERSECTION software including a lane-based platoon model for coordinated signals is discussed. The importance of the modelling of unequal lane use on external approaches of closely-spaced intersections is emphasised. This method coupled with a lane-based model allowing for the backward spread of congestion and upstream capacity constraint, as well as features such as short lane overflow, is expected to produce better results in assessing signal coordination quality and optimising signal offsets.

Further analyses of different lane use scenarios are recommended for their effects on signal platoon patterns and resulting performance estimates. Real-life surveys of lane use at closely-spaced intersections and analyses using microsimulation to compare results with those from analytical models are recommended.

\section{References}

[1] Akcelik and Associates. SIDRA INTERSECTION User Guide for Version 6.1. Akcelik and Associates Pty Ltd, Melbourne, Australia, 2015.

[2] Yumlu, C., Moridpour, S. and Akçelik, R. Measuring and Assessing Traffic Congestion: a Case Study. Paper presented at the AITPM Annual Meeting, Adelaide, Australia, 2014.

[3] Nicoli, F., Pratelli, A. and Akçelik, R. Improvement of the West road corridor for accessing to the New Hospital of Lucca (Italy). Paper to be published in Vol. 146 of WIT Transactions on the Built Environment, WIT Press, UK, 2015.

[4] Akçelik, R. Lane-based micro-analytical model of a roundabout corridor. CITE 2013 Annual Meeting, Calgary, AB, Canada, 2013. 
[5] Akçelik, R. Modelling Queue Spillback and Upstream Signal Effects in a Roundabout Corridor. TRB 4th International Roundabout Conference, Seattle, WA, USA, 2014.

[6] Akçelik, R. A New Lane-Based Model for Platoon Patterns at CloselySpaced Signalised Intersections. Paper presented at the 26th ARRB Conference, Sydney, 2014.

[7] Akçelik, R. Lane utilisation and saturation flows. Traffic Engineering and Control, 21(10), pp 482-484, 1980.

[8] Akçelik, R. Traffic Signals: Capacity and Timing Analysis. Research Report ARR No. 123. ARRB Transport Research Ltd, Vermont South, Australia, 1981 (6th reprint: 1995).

[9] Akçelik, R. SIDRA-2 does it lane by lane. Proc. 12th ARRB Conf. 12 (4), pp 137-149, 1984.

[10] Akçelik, R. On the estimation of lane flows for intersection analysis. Aust. Rd Res. 19(1), pp. 51-57, 1989.

[11] Akçelik, R. Lane-by-lane modelling of unequal lane use and flares at roundabouts and signalised intersections: the SIDRA solution. Traffic Engineering and Control, 38 (7/8), pp. 388-399, 1997.

[12] Taylor, W.C. and Abdel-Rahim, A.S. Analysis of Corridor Delay under SCATS Control (Orchard Lake Road Corridor). Final report. Department of Civil and Environmental Engineering, Michigan State University, East Lansing, Michigan, USA, 1998.

[13] TRB. Highway Capacity Manual. Transportation Research Board, National Research Council, Washington, DC, USA, 2010.

[14] Akçelik, R. Extension of the Highway Capacity Manual Progression Factor Method for Platooned Arrivals. Research Report ARR No. 276. ARRB Transport Research Ltd, Vermont South, Australia, 1995.

[15] Akçelik, R. Progression factor for queue length and other queue-related statistics. Transportation Research Record 1555, pp. 99-104, 1996.

[16] Akçelik, R. Green splits with priority to selected movements. Traffic Engineering and Control, 31 (7/8), pp. 402-405, 1990. 